\title{
Molecular Insight into the Slipperiness of Ice
}

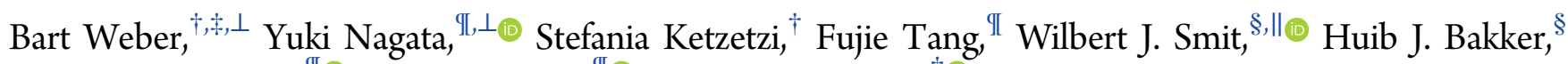

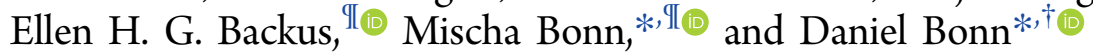

\author{
${ }^{\dagger}$ Van der Waals-Zeeman Institute, IoP, University of Amsterdam, Science Park 904, 1098XH Amsterdam, The Netherlands \\ IIMax Planck Institute for Polymer Research, Ackermannweg 10, 55128 Mainz, Germany \\ ${ }^{\S}$ AMOLF, Science Park 104, 1098 XG Amsterdam, The Netherlands
}

\section{Supporting Information}

\begin{abstract}
Measurements of the friction coefficient of steel-on-ice over a large temperature range reveal very high friction at low temperatures $\left(-100^{\circ} \mathrm{C}\right)$ and a steep decrease in the friction coefficient with increasing temperature. Very low friction is only found over the limited temperature range typical for ice skating. The strong decrease in the friction coefficient with increasing temperature exhibits Arrhenius behavior with an activation energy of $E_{\mathrm{a}} \approx 11.5 \mathrm{~kJ} \mathrm{~mol}^{-1}$. Remarkably, molecular dynamics simulations of the ice-air interface reveal a very similar activation energy for the mobility of surface molecules. Weakly hydrogen-bonded surface molecules diffuse over the surface in a rolling motion, their number and mobility increasing with increasing temperature. This correlation between macroscopic friction and microscopic molecular mobility indicates that slippery ice arises from the high mobility of its surface molecules, making the ice surface smooth and the shearing of the weakly bonded surface molecules easy.
\end{abstract}

S liding friction on ice is of paramount importance to a wide variety of subjects and phenomena including glaciology, ${ }^{1}$ automotive safety, ${ }^{2}$ frost heave, ${ }^{1}$ and winter sports. ${ }^{3}$ In each of these examples, ice surfaces can display an unusually low level of friction under a wide variety of conditions. Since the 19th century, this slipperiness of ice has continued to attract the attention of many scientists. ${ }^{4-6}$ A great variety of experimental and theoretical approaches have been deployed to elucidate the complex nature of sliding friction on ice, including but not limited to atomic force microscopy, ${ }^{7}$ sum-frequency generation vibrational spectroscopy, ${ }^{6}$ and molecular simulations. ${ }^{8}$ However, the precise molecular mechanism of the slipperiness remains unclear, despite a large number of studies dedicated to this important phenomenon. ${ }^{7-23}$

Here we perform macroscopic sphere-on-ice friction measurements that cover a larger range in temperature $\left(-100-0{ }^{\circ} \mathrm{C}\right)$ and sliding speed $\left(10^{-6}-10^{-1} \mathrm{~m} \mathrm{~s}^{-1}\right)$ than most studies to date. The main finding is that between -100 and $-10{ }^{\circ} \mathrm{C}$ the friction coefficient $(\mu)$, defined as the ratio of frictional to normal force, shows a strong Arrhenius-type temperature dependence, causing it to decrease by over an order of magnitude from values characteristic of normal solids $(\mu=0.5)$ to slippery values $(\mu=0.01)$. The activation energy for the Arrhenius behavior is the same as that for the surface diffusion of ice molecules. ${ }^{24}$ Our molecular dynamics (MD) simulations reveal the existence of two types of water at the topmost ice surface, one of which is very mobile; at high temperatures, this mobile water is enriched, accelerating the reorganization of the ice surface and making the surface smooth. Increasing the temperature, the friction decreases in a very similar manner to the inverse diffusion coefficient of the topmost water molecules on ice, suggesting that the sliding is facilitated by the topmost (mobile) surface ice molecules. At low temperatures, the surface mobility slows down and we observe strong velocity strengthening behavior, indicating that when the diffusion is too slow to accommodate the sliding motion friction increases.

We employ steel-on-ice sliding experiments over a large range of temperatures by using a $2.4 \mathrm{~mm}$ radius sphere-on-flat contact geometry; the use of a sphere allows us to estimate the contact area and penetration depth. The smooth stainless steel sphere is glued to a rheometer tool. By rotating the tool, the rheometer imposes sliding speeds for the sphere on the flat ice surface in the range of $10^{-6}-10^{-1} \mathrm{~m} \mathrm{~s}^{-1}$ while simultaneously measuring the normal, $N$, and frictional force, $F$ (Figure $1 \mathrm{~b}$ ). The friction coefficient $\mu=\frac{F}{N}$, the ratio of the frictional to the normal force, is as low as $\mu=0.01$ at temperatures around -7 ${ }^{\circ} \mathrm{C}$ (Figure 1a), a value similar to that obtained in actual ice skating. ${ }^{3}$ However, changing the temperature over a large range, we find a strong temperature dependence of $\mu$. A consequence of its dramatic increase at low temperatures is that at low

Received: April 16, 2018

Accepted: May 9, 2018

Published: May 9, 2018 
(a)

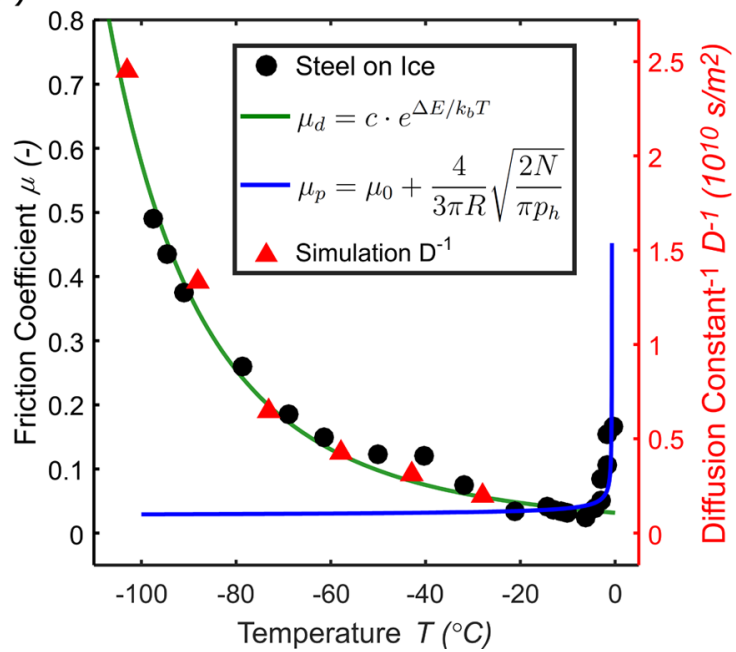

(b)

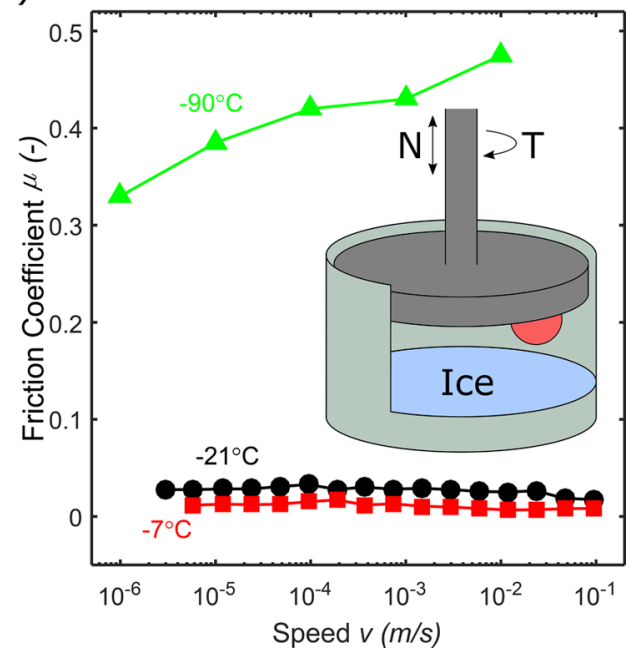

Figure 1. Steel-on-ice friction at different temperatures and speeds. (a) Steel-on-ice friction coefficient $\mu$ as a function of temperature measured at a constant sliding speed of $0.38 \mathrm{~mm} \mathrm{~s}^{-1}$ (black circles). Close to the melting point, the friction coefficient increases strongly, in agreement with a calculation of the friction coefficient resulting from plastic ploughing through the ice, using the temperature dependence of the penetration hardness of the ice, $p_{\mathrm{h}}$, obtained from indentation experiments (Supporting Information), a normal force $N=1 \mathrm{~N}$, and the surface friction contribution $\mu_{0}=$ 0.03 (blue line). At low temperatures, both the friction coefficient and the calculated inverse ice surface diffusion constant (red triangles) are found to follow Arrhenius temperature dependence with an activation energy of $\Delta E \approx 11.5 \mathrm{~kJ} \mathrm{~mol}^{-1}$ (green line). (b) Sliding speed dependence of the friction coefficient.

temperatures the ice surface is not slippery at all; the measured friction coefficient of $\mu=0.5$ at $-100{ }^{\circ} \mathrm{C}$ is typical for dry friction such as that between the same steel sphere and a glass surface (Figure S1).

The behavior of the friction coefficient as a function of temperature is in addition found to be nonmonotonic: from very low temperatures to roughly $-7{ }^{\circ} \mathrm{C}$, the friction coefficient steeply decreases, but above $-7{ }^{\circ} \mathrm{C}$, the friction coefficient rises sharply again (Figure 1a). This shows that there is an optimal temperature for sliding on ice of about $-7{ }^{\circ} \mathrm{C}$, which is indeed the temperature at which most skating rinks are kept to provide the fastest skating times. The sharp increase upon approaching the melting temperature can be rationalized as follows. Visual inspection of the ice surface shows that when the friction coefficient becomes high in this temperature range the sphere leaves a clearly visible ploughing track (Figure S2): the ice has plastically deformed. The sphere-on-flat geometry used here allows us to calculate the ploughing force based on the penetration hardness of the ice and the resulting ploughing cross section between the steel and the ice. The experimental friction coefficient for temperatures close to melting agrees well with this simple calculation (blue line in Figure $1 \mathrm{a}$ and Supporting Information). Furthermore, independent penetration experiments in which the sphere is pressed onto the ice surface and retracted again indeed show that at temperatures above $-7{ }^{\circ} \mathrm{C}$ the contact becomes fully plastic and the ice is irreversibly deformed (Figure S6).

Below $-7{ }^{\circ} \mathrm{C}$, the contact is purely elastic and the ice surface is not affected by the sliding. In this elastic regime, the friction force is proportional to the area of contact predicted by Hertzian $^{25,26}$ (elastic) contact mechanics and independent of the average contact pressure (Figures S1 and S2). Interestingly, the elastic properties of the ice do not vary greatly with temperature, ${ }^{27}$ and therefore, the contact area does not either. Consequently, because the friction force is proportional to the contact area, ${ }^{28}$ the change in friction observed at temperatures between -100 and $-7{ }^{\circ} \mathrm{C}$ must result from a change in the shear strength of the ice surface itself. ${ }^{11}$ Indeed, we find identical frictional behavior when we replace the steel sphere with a glass sphere (Figure S3). To investigate the nature of the change in shear strength, we vary the sliding velocity over more than 4 orders of magnitude (Figure 1b). Perhaps surprisingly, we find that in the low-friction regime the friction coefficient is independent of the sliding velocity. In the high-friction regime at low temperatures, however, we observe a marked velocity strengthening of the friction coefficient. Both results indicate that frictional heating does not play an important role in the experiments (Figure S9).

The macroscopic ice friction experiments suggest the presence of an ice surface layer that differs from the bulk and that somehow "lubricates" the contact as the temperature is increased from -100 to $-7{ }^{\circ} \mathrm{C}$. To obtain molecular-level information about this layer, we performed MD simulations using the POLI2VS model of water. ${ }^{29}$ As the lubrication mechanism is not affected ${ }^{30}$ by changes in the contact pressure or the use of different sliding materials, we investigate the iceair interface as the simplest model system for the ice surface.

The MD simulations reveal that the surface dynamics is governed by two distinct types of water molecules: ${ }^{31}$ molecules with one donating (D) and two accepting (A) hydrogen bonds (notation: DAA) and molecules donating and accepting one hydrogen bond (DA). In the simulations, DAA-type water molecules lose a hydrogen bond with increasing temperature, thereby changing from DAA to DA. The presence of DAA and DA water molecules on the ice surface and their temperature dependence predicted by theory are in quantitative agreement with those of SFG experiments (Figure S4). This interconversion of energetically stable DAA water to more weakly interacting and thus less stable DA molecules entails an increase in the mobility of water molecules across the surface; a reduced number of hydrogen bonds lowers the energetic barrier for translational motion. We quantify the lateral mobility of the surface molecules and link it to macroscopic ice friction using the time evolution of the in-plane two-dimensional (2D) 
(a)

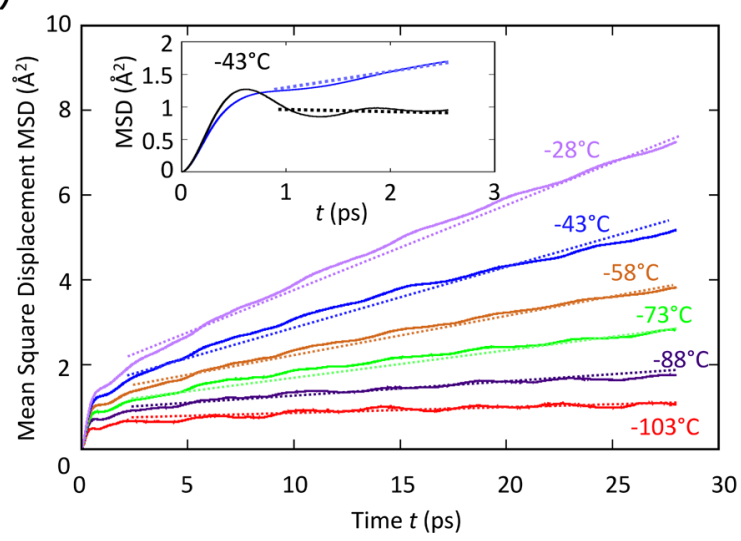

(b)

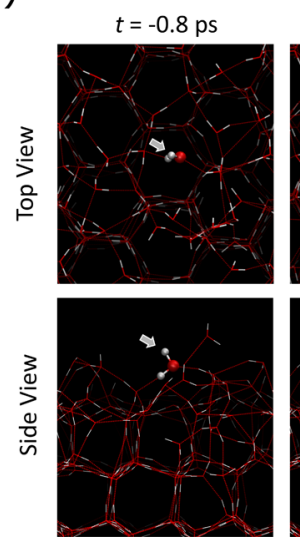

$t=0 \mathrm{ps}$
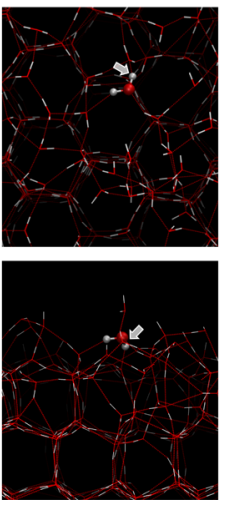
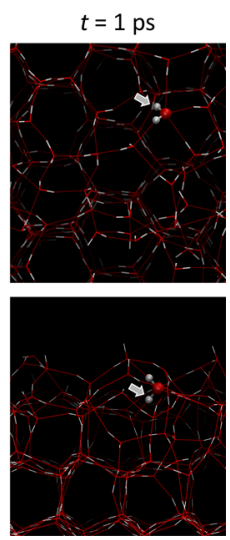

Figure 2. Mobility at the ice surface. (a) Calculated 2D MSD of the interfacial water molecules with free $\mathrm{O}-\mathrm{H}$ group(s) at different temperatures. Inset: comparison of the MSD of the interfacial water molecules that have free $\mathrm{O}-\mathrm{H}$ group (s) at time time 0 (blue) with that of the interfacial water molecules that keep free $\mathrm{O}-\mathrm{H}$ group(s) during time 0 to $t$ (black), both simulated at $-43{ }^{\circ} \mathrm{C}$. (b) Snapshots of the MD trajectory of the ice-air interface at $-43^{\circ} \mathrm{C}$. Top and bottom panels show the top and side views of the interface. The water molecule with the free $\mathrm{OH}$ group that forms the new hydrogen bond at positive $t$ is highlighted.

(a)

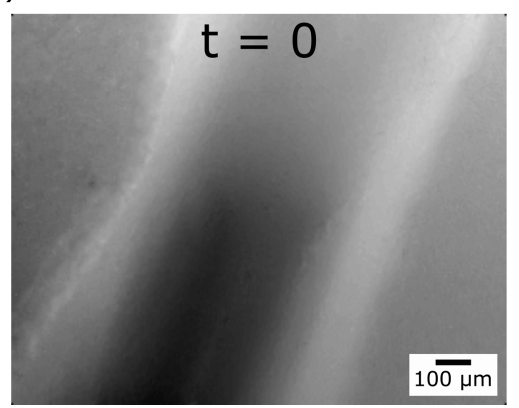

(b)

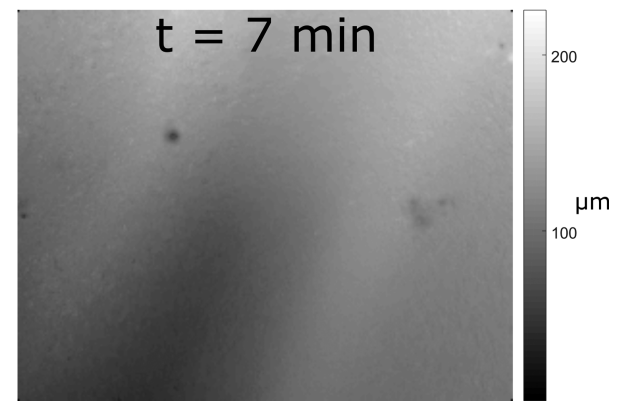

Figure 3. Self-healing ice at $-10{ }^{\circ} \mathrm{C}$. (a) Scratch profile measured with an interferometer just after a scratch was made manually on the ice surface using a screwdriver. (b) The scratch is almost completely healed just 7 min later.

mean-square displacement (MSD) of the DA water species at the topmost layer in the simulations

$$
\operatorname{MSD}(t)=\frac{\left\langle\theta(0)|r(t)-r(0)|^{2}\right\rangle}{\langle\theta\rangle}
$$

where $\theta(t)$ is 1 when a surface molecule has a free $\mathrm{O}-\mathrm{H}$ group at time $t$ and 0 otherwise. While heating the ice from -103 to $-28{ }^{\circ} \mathrm{C}$, we see a marked increase in the MSD of the surface molecules (Figure 2a).

The high mobility of the surface molecules has two important consequences for the slipperiness of ice. First, such a high mobility allows water molecules to fill in any defects on the ice surface very rapidly. We demonstrate this here by manually making a scratch into the ice surface and look at its evolution using a high-resolution interferometer (Figure 3). It can be observed that even a very deep scratch (made manually with a screwdriver) is filled in by the surface diffusion in a matter of minutes. This makes the surface very smooth; generally smooth surfaces are slipperier than their rough counterparts. Together with the observation that there is no ploughing at low temperature, this is a first indication of why ice is slippery. The thermally driven high lateral mobility of water molecules on an ice surface has been demonstrated previously: experiments on the sintering of ice spheres (the Faraday experiment) have shown that macroscopic quantities of ice molecules can be transported over millimeter length scales on a time scale of minutes, ${ }^{24}$ similar to our findings in this study. Furthermore, it has been established previously that surface molecules of solids can move at surprisingly high rates, and their dynamics has been compared to a giant atomic slide puzzle. $^{32}$ Taken together, these observations suggest that during the sliding motion on the ice shear is localized at these weakly bonded surface molecules and that their sliding ability determines the friction coefficient. If this were true, the friction coefficient should increase with increasing sliding speed whenever the mobility of the molecules becomes too low to follow the sliding motion. We observe this velocity strengthening effect in the low-temperature regime; the friction increases with increasing sliding speed (Figure 1b). Additionally, if surface mobility indeed facilitates the sliding, we would expect an inverse relation between friction and mobility: the higher the mobility, the lower the friction. This is exactly what we observe; in Figure 1a, where we plot the inverse diffusion coefficient, $D^{-1}(T)$

$$
D=\lim _{t \rightarrow \infty} \frac{\left\langle\theta(0)|r(t)-r(0)|^{2}\right\rangle}{4 t\langle\theta\rangle}
$$

together with the friction coefficient as a function of temperature.

Friction and mobility follow the same Arrhenius temperature dependence with an activation energy of $\Delta E \approx 11.5 \mathrm{~kJ} \mathrm{~mol}^{-1}$ 
(Figure 1a), almost half of the hydrogen-bond energy of $21 \mathrm{~kJ}$ $\mathrm{mol}^{-1}$.

The MD trajectories displayed in Figure $2 \mathrm{~b}$ demonstrate that a DA water molecule at the ice surface moves by exchanging its hydrogen bond partner (Supporting Inormation movie). In fact, the MSD for a DA molecule which keeps free $\mathrm{O}-\mathrm{H}$ group(s) does not show clear diffusion, as is seen in Figure 2a inset. This exchange occurs by breaking the hydrogen bonds of the topmost water molecules. As is evident from the SFG spectra of the free $\mathrm{O}-\mathrm{H}$ stretch peak (Figure S4), by increasing the temperature, the hydrogen bond tends to be broken, converting DAA water molecules to DA water molecules and increasing the surface mobility. During frictional sliding, we submit that these weakly bonded surface molecules determine the friction coefficient. We therefore conclude that at temperatures close to melting ice is slippery due to highly mobile DA water molecules that diffuse over the ice surface in a rolling motion through exchange of the hydrogen bond partner: there is one molecular layer of mobile water at the surface that lubricates the contact.

In summary, combined macroscopic sliding friction measurements and MD simulations reveal that the macroscopic friction coefficient of ice and the microscopic mobility of the topmost water molecules on the ice surface exhibit very similar temperature dependence. Quantitatively, both exhibit an activation energy of $\Delta E \approx 11.5 \mathrm{~kJ} \mathrm{~mol}^{-1}$, suggesting that friction is correlated with the presence of the mobile DA water molecules at the topmost ice surface. This would imply that ice is slippery due to highly mobile DA water molecules that diffuse over the ice surface: there is a layer of mobile ice at the surface that makes the surface smooth and lubricates the contact. The simulations show that the fraction of mobile molecules strongly increases with increasing temperature. This then allows the ice to evolve from a normal solid surface at low temperatures to a very slippery surface close to the melting temperature. While the presence of a quasi-liquid layer on ice has often been held responsible for the slipperiness of ice, our results indicate that surface melting contributes indirectly to slipperiness by creating highly mobile surface molecules. We note, however, that the friction coefficient varies smoothly with temperature, and no discontinuities are observed that could be attributed to discrete surface melting transitions.

It is perhaps not so surprising that the energetic barrier for translational motion on the surface is directly relevant for friction; ${ }^{33}$ skating on Teflon skating rinks for example is thought to be possible because the activation energy for the slippage between crystalline Teflon slices is low, causing high wear and low friction on Teflon. ${ }^{34}$ In friction at the nanoscale, the energy barrier for translational motion of an AFM tip over a surface can become small compared to the thermal energy, resulting, again, in very low friction; temperature acts as a lubricant because the energy required to locally move from one potential minimum to the next is supplied thermally. ${ }^{35}$ Furthermore, mobile hydration shells consisting of water molecules surrounding ions are conjectured to cause low friction between such hydrated ions. ${ }^{36}$

\section{ASSOCIATED CONTENT}

\section{(S Supporting Information}

The Supporting Information is available free of charge on the ACS Publications website at DOI: 10.1021/acs.jpclett.8b01188.
Normal force dependence of the friction force, glass-onice friction measurements, SFG experiments, friction experiments on monocrystalline ice, the ploughing model, ice stiffness and hardness, frictional heating, and details of the MD simulations (PDF)

Movie showing molecule movement at the ice surface (WMV)

\section{AUTHOR INFORMATION}

\section{Corresponding Authors}

*E-mail: bonn@mpip-mainz.mpg.de (M.B.).

*E-mail: d.bonn@uva.nl (D.B.).

ORCID 을

Yuki Nagata: 0000-0001-9727-6641

Wilbert J. Smit: 0000-0002-0543-7258

Ellen H. G. Backus: 0000-0002-6202-0280

Mischa Bonn: 0000-0001-6851-8453

Daniel Bonn: 0000-0001-8925-1997

\section{Present Addresses}

${ }^{\ddagger}$ B.W.: Advanced Research Center for Nanolithography (ARCNL), Science Park 110, 1098 XG Amsterdam, Netherlands.

"W.J.S.: ESPCI Paris, PSL Research University, UMR CBI 8231, 10 rue Vauquelin, 75005 Paris, France.

\section{Author Contributions}

${ }^{\perp}$ B.W. and Y.N. contributed equally.

\section{Notes}

The authors declare no competing financial interest.

\section{ACKNOWLEDGMENTS}

The authors thank Hans van Leeuwen, Sander Woutersen, Joost Frenken, Tjerk Oosterkamp, and Sergey Krylov for very helpful discussions. This work is part of the FOM-Programme Fundamental Aspects of Friction, financed by FOM/NWO.

\section{REFERENCES}

(1) Dash, J. G.; Rempel, A. W.; Wettlaufer, J. S. The Physics of Premelted Ice and Its Geophysical Consequences. Rev. Mod. Phys. 2006, 78, 695-741.

(2) Strong, K. C.; Ye, Z.; Shi, X. Safety Effects of Winter Weather: The State of Knowledge and Remaining Challenges. Transp. Rev. 2010, 30, 677-699.

(3) de Koning, J. J.; de Groot, G.; van Ingen Schenau, G. J. Ice Friction During Speed Skating. J. Biomech. 1992, 25, 565-571.

(4) Joly, J. Sci. Proc. R. Soc. Dublin 1886, 5, 453.

(5) Rosenberg, R. Why is Ice Slippery? Phys. Today 2005, 58, 50-55.

(6) Li, Y.; Somorjai, G. A. Surface Premelting of Ice. J. Phys. Chem. C 2007, 111, 9631-9637.

(7) Döppenschmidt, A.; Kappl, M.; Butt, H.-J. Surface Properties of Ice Studied by Atomic Force Microscopy. J. Phys. Chem. B 1998, 102, 7813-7819.

(8) Gladich, I.; Pfalzgraff, W.; Marsalek, O.; Jungwirth, P.; Roeselová, M.; Neshyba, S. Arrhenius Analysis of Anisotropic Surface SelfDiffusion on the Prismatic Facet of Ice. Phys. Chem. Chem. Phys. 2011, 13, 19960-19969.

(9) Gurney, C. Surface Forces in Liquids and Solids. Proc. Phys. Soc., London, Sect. A 1949, 62, 639.

(10) Barnes, P.; Tabor, D.; Walker, J. C. F. The Friction and Creep of Polycrystalline Ice. Proc. R. Soc. London, Ser. A 1971, 324, 127-155.

(11) Persson, B. N. J. Ice Friction: Role of Non-Uniform Frictional Heating and Ice Premelting. J. Chem. Phys. 2015, 143, 224701.

(12) Kvlividze, V. I.; Kiselev, V. F.; Kurzaev, A. B.; Ushakova, L. A. The Mobile Water Phase on Ice Surfaces. Surf. Sci. 1974, 44, 60-68. 
(13) Golecki, I.; Jaccard, C. Intrinsic Surface Disorder in Ice Near the Melting Point. J. Phys. C: Solid State Phys. 1978, 11, 4229.

(14) Engemann, S.; Reichert, H.; Dosch, J.; Bilgram, V.; Honkimäki, V.; Snigirev, A. Interfacial Melting of Ice in Contact With $\mathrm{SiO}_{2}$. Phys. Rev. Lett. 2004, 92, 205701.

(15) Sánchez, M. A.; et al. Experimental and Theoretical Evidence for Bilayer-by-Bilayer Surface Melting of Crystalline Ice. Proc. Natl. Acad. Sci. U. S. A. 2017, 114, 227-232.

(16) Kietzig, A.-M.; Hatzikiriakos, S. G.; Englezos, P. Physics of Ice Friction. J. Appl. Phys. 2010, 107, 081101-081101.

(17) Bowden, F. P.; Hughes, T. P. The Mechanism of Sliding on Ice and Snow. Proc. R. Soc. London, Ser. A 1939, 172, 280-298.

(18) van Leeuwen, J. M. J. Skating on Slippery Ice. SciPost Phys. 2017, 3, 042 .

(19) Ovaska, M.; Tuononen, A. J. Multiscale Imaging of Wear Tracks in Ice Skate Friction. Tribol. Int. 2018, 121, 280-286.

(20) Liang, H.; Martin, J. M.; Mogne, T. L. Experimental Investigation of Friction on Low-Temperature Ice. Acta Mater. 2003, 51, 2639-2646.

(21) Colbeck, S. C.; Najarian, L.; Smith, H. B. Sliding Temperatures of Ice Skates. Am. J. Phys. 1997, 65, 488-492.

(22) Fowler, A. J.; Bejan, A. Contact Melting During Sliding on Ice. Int. J. Heat Mass Transfer 1993, 36, 1171-1179.

(23) Wei, X.; Miranda, P. B.; Shen, Y. R. Surface Vibrational Spectroscopic Study of Surface Melting of Ice. Phys. Rev. Lett. 2001, $86,1554$.

(24) Kingery, W. D. Regelation, Surface Diffusion, and Ice Sintering. J. Appl. Phys. 1960, 31, 833-838.

(25) Hertz, H. Über die Berührung Fester Elastischer Körper. J. Reine Angew. Math. 1882, 92, 156-171.

(26) Suhina, T.; Weber, B.; Carpentier, C. E.; Lorincz, K.; Schall, P.; Bonn, D.; Brouwer, A. M. Fluorescence Microscopy Visualization of Contacts Between Objects. Angew. Chem., Int. Ed. 2015, 54, 36883691.

(27) Butkovich, T. Strength Studies of Sea Ice; Snow Ice and Permafrost Research Establishment, Corps of Engineers, U.S. Army, 1956.

(28) Weber, B.; Suhina, T.; Junge, T.; Pastewka, L.; Brouwer, A. M.; Bonn, D. Molecular Probes Reveal Deviations From Amontons' Law in Multi-asperity Frictional Contacts. Nat. Commun. 2018, 9, 888.

(29) Hasegawa, T.; Tanimura, Y. A Polarizable Water Model for Intramolecular and Intermolecular Vibrational Spectroscopies. J. Phys. Chem. B 2011, 115, 5545-5553.

(30) Dhopatkar, N.; Defante, A. P.; Dhinojwala, A. Ice-like Water Supports Hydration Forces and Eases Sliding Friction. Sci. Adv. 2016, 2, e1600763.

(31) Smit, W. J.; Tang, F.; Sánchez, M. A.; Backus, E. H. G.; Xu, L.; Hasegawa, T.; Bonn, M.; Bakker, H. J.; Nagata, Y. Excess Hydrogen Bond at the Ice-Vapor Interface around 200 K. Phys. Rev. Lett. 2017, 119, 133003.

(32) van Gastel, R.; Somfai, E.; van Saarloos, W.; Frenken, J. A Giant Atomic Slide-Puzzle. Nature 2000, 408, 665.

(33) Louden, P. B.; Gezelter, J. D. Friction at Ice-Ih/Water Interfaces is Governed by Solid/Liquid Hydrogen-Bonding. J. Phys. Chem. C 2017, 121, 26764-26776.

(34) Tanaka, K.; Uchiyama, Y.; Toyooka, S. The Mechanism of Wear of Polytetrafluoroethylene. Wear 1973, 23, 153-172.

(35) Jinesh, K. B.; Krylov, S. Yu.; Valk, H.; Dienwiebel, M.; Frenken, J. W. M. Thermolubricity in Atomic-Scale Friction. Phys. Rev. B: Condens. Matter Mater. Phys. 2008, 78, 155440.

(36) Ma, L.; Gaisinskaya-Kipnis, A.; Kampf, N.; Klein, J. Origins of Hydration Lubrication. Nat. Commun. 2015, 6, 6060. 